\title{
Ensino de bioética em cursos superiores de computação: uma análise crítica
}

\author{
Rodrigo Cândido Borges ${ }^{1}$, Maria Márcia Bachion² \\ ${ }^{1}$ Departamento de Áreas Acadêmicas - Instituto Federal de Goiás (IFG) \\ Inhumas - GO - Brasil \\ ${ }^{2}$ Faculdade de Enfermagem - Universidade Federal de Goiás (UFG) \\ Goiânia - GO - Brasil \\ rodrigocandegmail.com, mbachionegmail.com
}

\begin{abstract}
Since its inception, bioethics has gone through different phases and one of the best known is clinical bioethics. However, its original scope is much broader and is being resumed, which allows it to approach more diversified areas. In the present article, we try to highlight themes and dimensions of Computer Science that lack bioethical reflections and the reader is invited to the dialogue about the possibility of insertion of Bioethics in the curriculum of the superior courses of computation.
\end{abstract}

Resumo: Desde o seu surgimento, a bioética passou por diferentes fases e uma das mais conhecidas é a bioética clínica. Contudo, seu escopo original é bem mais amplo e está sendo retomado, o que permite sua aproximação com áreas bastante diversificadas. No presente artigo, busca-se evidenciar temas e dimensões da Ciência da Computação que carecem de reflexões bioéticas e convida-se o leitor para o diálogo sobre a possibilidade de inserção da Bioética no currículo dos cursos superiores de computação.

\section{Introdução}

Os avanços científicos e tecnológicos advindos da evolução computacional possibilitaram significativas transformações nas interações sociais, na comunicação, no acesso e na difusão da informação. $\mathrm{O}$ impacto dessas inovações requer discussões acerca de suas implicações, seus danos e benefícios, bem como diálogo sobre suas consequências éticas e bioéticas.

A compreensão que a ciência resulta em mais tecnologia, ganho capital e bemestar social tem sido questionada, pois as linhas tecnológicas não são equânimes e possuem implicações e usos que também precisam ser refletidos (Roso e Auler, 2016).

Com um escopo pluralista e reflexivo (Potter, 1970), a bioética integra saberes científicos e morais, o conhecimento e os valores humanos, transcendendo mais do que o mero plano das normas e dos códigos de ética.

A preocupação com formação em Bioética por meio de sua incorporação nos currículos em nível de graduação ocorreu inicialmente na área de saúde (Neves Júnior, Araújo e Rego, 2016; Maluf e Azambuja, 2015; Couto Filho et al., 2013). Gradativamente, áreas como as Ciências Biológicas passaram a perceber a necessidade de incluir o tema na formação dos futuros profissionais (Dória e Moreira, 2011). 
No entanto, isso pouco tem sido explorado em outras graduações que, embora não sejam da área da saúde, atuam direta ou indiretamente em contextos nos quais são inerentes questões e dilemas envolvendo o bem-estar, a felicidade e a segurança de seres humanos.

A computação é geradora de tecnologias variadas e revolucionárias, capazes de promover a qualidade de vida da humanidade, mas, também, com possibilidades de comprometer o ambiente, as relações sociais e desvalorizar determinadas culturas. Promover reflexões bioéticas, no processo de construção do conhecimento daqueles que estarão envolvidos no desenvolvimento científico, torna-se relevante em um contexto onde suas ações sempre são repletas de consequências para a vida humana.

O presente trabalho tece uma análise crítica que contempla especialmente a inclusão da bioética no currículo de formação superior dos profissionais ligados à informática, abordando realidades, iniciativas e contestações.

\section{A Bioética de Potter}

Van Rensselaer Potter, em 1970, divulgou seu primeiro texto utilizando a palavra Bioética (do inglês Bioethics). O artigo intitulado Bioethics, the science of survival destacava a necessidade de uma ética abrangente, aliando os saberes científico e moral (Potter, 1970). Em 1971, publicou o livro Bioethics: A Bridge to the Future, no qual definiu bioética como ponte entre ciências e humanidades (Potter, 1971). Os questionamentos levantados pelo autor apoiavam-se na preocupação com um futuro dito duvidoso, sobretudo devido à falta de diálogo entre os saberes científicos e os princípios éticos.

Em 1988, foi publicada sua segunda obra, Global Bioethics: Building on the Leopold Legacy (Potter, 1988), em que ampliou a bioética a outras disciplinas, não se restringindo à ponte entre a biologia e a ética, mas com a dimensão de uma Ética Global. Nesta concepção, estão envolvidos todos os aspectos relativos ao viver, o conhecimento biológico, a ciência dos sistemas vivos, os valores humanos e a ética médica.

Posteriormente, em 1998, propôs a definição da chamada Bioética Profunda (Potter, 1998), uma nova ciência ética apoiada na humildade, responsabilidade, competência intercultural e interdisciplinar, potencializando o senso de humanidade. Este conceito evidencia seu esforço em manter características pluralistas para incorporação de novos conhecimentos, centrado no pensamento de que a possibilidade de assumir erros promove aberturas para aprender com novas experiências.

Na sua origem, com Potter (1970), a Bioética não se propunha a ser uma teoria que pudesse nortear o processo de análise, julgamentos e tomadas de decisão. Contudo, com o fortalecimento da Bioética Clínica, após as concepções de Beauchamp e Childress (2002) desenvolvidas mediante debates suscitados por pesquisas moralmente questionáveis na área médica, e o enfrentamento de dilemas cada vez mais frequentes na área da saúde, desenvolveram-se modelos de tomada de decisão baseados em princípios éticos (Loch, 2003). Para Otero (2009), esse movimento representou uma usurpação, uma dissidência do sentido do neologismo originalmente proposto por Potter (1970, 1971), opinião corroborada por Pessini (2013). Segundo Cunha e Lorenzo (2014), a partir de 2005 ampliaram-se os horizontes da Bioética na comunidade científica, após a 
publicação da Declaração Universal sobre Bioética e Direitos Humanos pela Organização das Nações Unidas para a Educação, a Ciência e a Cultura (Unesco).

Pessini (2013) destaca trechos da fala de Potter (1998) nos quais se percebe seu chamamento para que se compreenda a "bioética como uma nova ética científica que combina humildade, responsabilidade e competência, numa perspectiva interdisciplinar e intercultural que potencializa o sentido de humanidade" e a transforme em uma disciplina (entendida como um campo de conhecimento científico).

Assim, percebe-se que a Bioética é um campo dinâmico, ao mesmo tempo controverso, mas que não se pode ignorar. Cada grupo de estudiosos pode adotar um sentido específico (Cunha e Lorenzo, 2014), mas com um ponto em comum: a reflexão filosófica sobre o modo de vida e o modo de relação dos seres humanos entre si, entre eles e os outros seres vivos, entre eles e o ambiente.

Segundo Caramico, Zaher e Rosito (2007), na visão de Potter, a bioética representa uma amplitude que contempla o conhecimento científico e filosófico. Para eles, a bioética foi criada para nortear avanços tecnológicos em todas as áreas, em prol da humanidade, sendo de interesse da maioria da sociedade.

O pensar Bioética deve se estender a aspectos do cotidiano dos profissionais e, de modo geral, dos cidadãos, o que demanda processos de formação. Na perspectiva da educação, é necessária a abertura para novos conteúdos, a fim de que os alunos pensem moralmente por si próprios e decidam com autonomia (Neves Júnior, Araújo e Rego, 2016).

\section{O Ensino de Bioética em Ciências da Saúde}

Questões éticas estão historicamente presentes na Medicina desde seus primórdios, o que pode ser constatado no juramento solene de Hipócrates, escrito há mais de 2 mil anos (Carneiro et al., 2010). Contudo, segundo Caramico, Zaher e Rosito (2007), a ética médica alcançou um novo patamar após o surgimento da Bioética e de sua inclusão no ensino de graduação nessa profissão, assim como abriu horizontes, protocolou temas para reflexão, expandiu e modificou tratamentos médicos, trouxe o imperativo de mais respeito para com o outro e inter-relacionou especialidades médicas e não médicas. Após a análise de 76 projetos pedagógicos de cursos de Medicina do Brasil, a pesquisa realizada por Neves Júnior, Araújo e Rego (2016) identificou que 25 faculdades apresentavam disciplinas com conteúdos de bioética associados a outras matérias; enquanto 31 possuíam em sua matriz curricular disciplinas autônomas com conteúdo e nome "Bioética".

Apesar de importante, a inserção de formação em bioética na Medicina não deve se limitar à dimensão curricular. O trabalho de Camargo, Almeida e Morita (2014) revelou que a maioria dos alunos do sexto ano da graduação considera este um tema importante, mas não acompanha as discussões que o envolvem nos espaços extracurriculares.

A Bioética como componente do currículo também se faz presente em faculdades de Odontologia brasileiras. Maluf e Azambuja (2015) enfatizam que o dentista realiza atividades clínicas, cirúrgicas, administra recursos humanos e materiais, mas suas relações com os pacientes ainda são permeadas por conflitos e dilemas que exigem atenção e preparo moral, ético e bioético para contorná-los ou preveni-los. 
Valladão et al. (2011) ressaltam que, na ciência odontológica contemporânea, os problemas éticos podem surgir tanto na graduação como na pós-graduação e na clínica odontológica, assim como se entrelaçar e se sobrepor, o que evidencia a importância da difusão da bioética para a comunidade e universidade.

De acordo com Mascarenhas e Rosa (2010), o ensino da Bioética na graduação em Enfermagem deve se adequar às demandas para a formação de um profissional generalista, humanista, crítico, reflexivo e essencialmente ético, voltado para atender às necessidades de saúde, nos âmbitos individual e coletivo. Couto Filho et al. (2013) investigaram a oferta da disciplina ou da temática bioética nos cursos de graduação em Enfermagem das universidades federais brasileiras. Das 16 instituições que atendiam ao critério de inclusão do estudo, em oito delas $(50 \%)$ a Bioética era apresentada como temática específica ou constava na grade curricular do curso. Os autores consideram que não inserir este tema na formação impõe prejuízos aos futuros profissionais.

\section{A Inserção da Bioética na Formação de Recursos Humanos}

No âmbito da educação superior brasileira, os cursos pertencentes às Ciências da Saúde se destacam no pioneirismo da oferta desta disciplina. Com o passar dos anos, outras áreas do conhecimento têm incluído o ensino da Bioética na composição programática de seus cursos.

No campo das Ciências Biológicas, pesquisa realizada por Dória e Moreira (2011) explorou a inserção curricular da Bioética (ou similares) nos cursos de Biologia de 36 Instituições Federais de Ensino Superior no Brasil. Segundo o estudo, 50\% das universidades analisadas tinham instituído o conteúdo e trabalhavam, sobretudo, temas relacionados à ética biomédica com uma notável abordagem globalizante, incluindo questões socioambientais e ecológicas. Siebert (2015) assinala alguns dilemas bioéticos que incidem sobre a formação do biólogo: questões voltadas ao consumismo, poluição, recursos energéticos, crescimento demográfico, alimentos transgênicos e manipulações genéticas. Para este autor, o ensino de bioética é indispensável nos cursos de formação de profissionais ligados à área de ciências biológicas, sobretudo devido à desarmonia existente entre os avanços tecnológicos e as reflexões morais sobre suas consequências.

Ao analisar a formação inicial de professores de Ciências e Biologia, Silva e Krasilchik (2013) puderam verificar lacunas na formação inicial dos docentes para lidarem com fatos que demandem mediação de discussões ou tomada de posição. Os autores frisam a importância da bioética no debate de alguns temas principais, tais como limites das atividades científicas, preservação ecológica, soberania geopolítica, consentimento e autonomia, que deveriam ser obrigatoriamente abordados durante a formação inicial de professores de Ciências e Biologia, a fim de prepará-los para essas discussões com os alunos.

A inserção de bioética é crescente também nas Ciências Agrárias. A Medicina Veterinária tem se destacado por tornar a disciplina obrigatória em algumas instituições, tais como a Universidade Federal de Goiás, Universidade Federal de Uberlândia, Universidade do Estado da Bahia, entre outras. Questões importantes como o bem-estar animal e a biossegurança estão inseridas em diversos ementários, sendo também objeto de debates em congressos específicos (CFMV, 2018). Para Zanetti (2009), conhecer os elementos que compõem as experiências dos alunos nas aulas práticas em que animais são usados como recurso didático é um desafio educacional, pois essas vivências afetam 
a construção da formação profissional e podem ser consideradas expressão de violência ou crueldade contra os animais e também contra os próprios alunos.

No campo das Ciências Sociais Aplicadas, as Faculdades de Direito têm se sobressaído na inserção curricular de Bioética. Novas abordagens metodológicas vislumbram familiarizar os estudantes com uma nova compreensão da ciência jurídica, correlacionando-a com as ciências da vida e conferindo sentido ao chamado Biodireito (Pereira, 2015). Villas-Bôas (2012) salienta que o cabedal epistemológico desenvolvido pela bioética nos últimos anos traz um ganho para o Direito e para a sociedade. A aproximação dos profissionais do âmbito jurídico e de saúde tende a enriquecer esses diálogos e contribuir para o crescimento e empoderamento do ser humano.

Em relação às Engenharias, Adinolfi (2014) conclui que a Bioética e a educação em valores configuram-se como uma área ainda silenciosa, uma lacuna caracterizada pela ausência de materiais, disciplinas e conteúdos nas grades curriculares dos cursos de graduação. Segundo a autora, o desconhecimento e o desinteresse pela bioética podem decorrer do aspecto tecnicista da formação de engenheiros no Brasil, cujo foco é determinar a exequibilidade de um projeto, dando pouca ênfase à sua viabilidade e ao quanto ele é, ou não, desejável. Dentre 88 cursos de graduação em Engenharia do estado de São Paulo analisados, apenas um incluía a Bioética; outros 12 tinham conteúdos de formação ética em geral (Adinolfi, 2014).

No tocante às Ciências Exatas e da Terra, não foram encontrados indícios de inserção da Bioética na matriz curricular em cursos superiores.

A baixa inclusão ou ausência da Bioética como disciplina em áreas fora do âmbito da saúde suscita uma série de indagações sobre o perfil do egresso de grande parte dos cursos de graduação brasileiros. O modelo educacional tradicionalmente adotado volta-se, em sua maioria, à formação técnica dos discentes, pouco contemplando a formação de valores e o olhar sobre outros campos do conhecimento. A educação como doutrinação dificulta a oferta de abordagens multidisciplinares como a de Bioética, que remetem à reflexão de assuntos relacionados aos impactos científicos e tecnológicos, além dos dilemas que afetam os rumos e a sobrevivência do planeta.

\section{Bioética e Computação}

A computação é a ciência que se dedica ao tratamento da informação mediante o uso de computadores ou dispositivos que permitem o processamento de dados. Com a inclusão digital, os computadores, inicialmente restritos às grandes organizações, passaram a integrar o contexto doméstico. A preocupação em fazer com que as máquinas e seus conteúdos fossem acessíveis a todos foi um fator primordial para a popularização das tecnologias e motivou investimentos para linhas como as arquiteturas de computadores, engenharia de software, redes de computadores, computação móvel e ubíqua, entre outras (Carvalho, 2006).

Os ganhos promovidos pelas tecnologias informáticas contribuem para o desenvolvimento científico não só das Ciências Exatas, mas também de outras áreas dos saberes, consistindo em um ramo de pesquisa altamente aplicável que objetiva atender a diferentes anseios. No contexto da saúde, métodos avançados de inteligência artificial, como o aprendizado profundo, têm sido aplicados no desenvolvimento de programas que simulam as atividades dos neurônios da região do cérebro onde ocorrem os 
pensamentos. Outras frentes caminham para a ideia de supercomputadores contribuírem no diagnóstico de enfermidades, utilizando a alta capacidade de armazenamento de dados aliados a algoritmos inteligentes (Siuly, Huang e Daneshmand, 2018). No campo da Bioética, redes neurais artificiais têm sido modeladas para que as deliberações clínicas sejam acertadas do ponto de vista tanto técnico quanto ético (Siqueira-Batista et al., 2014).

Quanto às soluções voltadas aos usuários de dispositivos móveis, o aplicativo "Meu digiSUS" (MS, 2018), criado pelo Departamento de Informática do SUS, é um exemplo do que se pode oferecer em relação a informações em saúde de uso pessoal e restrito aos cidadãos, como o acesso a dados do cartão nacional de saúde, listas de exames realizados e de medicamentos retirados e acompanhamento do cartão de vacinação.

Apesar das notórias cooperações da computação com outras áreas na contemporaneidade, algumas de suas derivações científicas e tecnológicas são objetos de questionamentos. O mercado de software pirata é um caso típico, alimentado por um consumismo desenfreado de parte da população, mantenedora de um cenário ilícito. A empresa Business Software Alliance (BSA, 2018) relata que cerca da metade dos programas instalados em computadores no Brasil são ilegais. Ao longo de 2016, só na América Latina, foram gastos 5,8 bilhões de dólares com programas piratas.

O uso de programas piratas traz prejuízos diretos para as empresas proprietárias das tecnologias originais. A lucratividade retirada das organizações de forma ilegal compromete os planos de gestão e negócios, lesando, por exemplo, a possibilidade de contratação de funcionários, ou mesmo interferindo nos gastos destinados a possíveis melhorias dos sistemas. Trata-se de um dano que envolve condutas antiéticas tanto por parte de usuários como de profissionais de informática, disseminadores deste tipo de produto.

A necessidade de preservar a integridade, confidencialidade, disponibilidade e autenticidade dos dados digitais faz com que a segurança da informação seja uma disciplina de destaque na computação moderna. O grande dilema reside em definir quem tem direito a acessá-los e alterá-los. Uma informação, ao ser considerada confidencial, traz uma série de implicações, por extrapolar a esfera técnica e atingir a esfera ética (Mann, 2011).

Em 2017, uma série de ataques cibernéticos ocorreu em computadores de hospitais e empresas de comunicação, em pelo menos 70 países. Na Inglaterra, médicos de hospitais públicos tentavam acessar os prontuários de pacientes e se defrontavam com avisos de bloqueio das informações e liberação somente após o pagamento de um resgate. Tratava-se de um sequestro de dados digitais, uma modalidade de crime denominado ransomware (ANSA, 2018).

Outro fato polêmico consiste na autonomia e privacidade em ambientes corporativos. Programas têm sido desenvolvidos e implantados para monitorar imagens de tela, movimentos do mouse e sinais do teclado dos funcionários em empresas, o que tem incrementado debates sobre o limite de um software. Defensores da causa argumentam que o computador da empresa não é ferramenta dos funcionários. Assim, ver notícias, fazer compras ou realizar download de jogos e aplicativos são ações que deveriam ser executadas apenas em casa, sob risco de demissão. Outra frente defende 
que tal prática denota invasão de privacidade, por ferir princípios da liberdade e legalidade (Sitesa, 2018).

O impacto ambiental da computação também é motivo de controvérsias. Os equipamentos eletrônicos são considerados sucatas, geralmente em curto prazo, despejando anualmente milhões de toneladas deste tipo de lixo, composto por uma série de materiais, alguns altamente tóxicos, como o chumbo, o mercúrio e o cádmio. Além disso, o consumo energético de equipamentos eletrônicos, na atual era da internet e das telecomunicações, é da ordem de bilhões de quilowatts-hora (Farinaccio, 2016). Essa série de questões demanda cada vez mais dos profissionais da área de computação, desde a formação, uma postura ética e de colaboração em relação à sociedade, à vida e ao meio ambiente.

Esses profissionais encontram-se cada vez mais envolvidos em decisões de projetos, avaliando o grau de confiabilidade e tolerância a falhas de sistemas (Raymond, 2015). A quase totalidade dos programas é gerenciada por usuários que têm acesso a informações confidenciais ou críticas. Portanto, a formação discente deve envolver habilidades que transcendam a capacidade prática e alcancem a reflexão das consequências. A instituição de ensino superior que não prepara seus alunos para atender a este novo quadro não o capacita para um mercado tão inovador.

O mundo para qual o ensino superior forma os profissionais tem mudado e impõe novas demandas às instituições. $\mathrm{O}$ cenário econômico globalizado alterou as fronteiras do conhecimento, da cultura e do mercado (Adinolfi, 2014). As tecnologias da informação e comunicação geraram uma nova linguagem, icônica e global. A era do conhecimento individual, baseado na cultura impressa, foi substituída por um tempo em que o conhecimento é uma construção coletiva, apreendido pela cultura digital (Adinolfi, 2014).

Segundo Camargo, Almeida e Morita (2014), os alunos representam um campo fértil, pois anseiam por atendimento humanitário e questionam realidades maiores, aspecto que permite inferir interesse e capacidade de absorver a abordagem da ética e da bioética na graduação.

Essa inserção poderia ocorrer sob forma de uma disciplina ou tema transversal, ambas com seus prós e contras. Como disciplina, pode constituir uma fortaleza quando desenvolvida por docentes com perfil adequado, estudiosos do campo específico e capazes de relacionar de forma dinâmica a Bioética e a Ciência da Informação, mas há risco do ensino tornar-se um processo delimitado no tempo e espaço, sem aplicação em outros momentos da formação. Para superar este aspecto, sua inserção no Projeto Pedagógico do Curso deve privilegiar seu entendimento como tema transversal. Se incluída em outras disciplinas, como Informática e Sociedade, por exemplo, pode perder a sua centralidade, a sua essência, em meio a outros temas discutidos. Por outro lado, existir ou fortalecer-se neste espaço é possível mediante a participação de especialistas e uso de abordagens que favoreçam a aprendizagem significativa. Não há um caminho exclusivo, pois as alternativas podem se somar, alternar, até que se encontre uma proposta que permita a criação de um espaço para que o diálogo entre a Bioética e a Ciência da Computação, na graduação, propicie a formação diferenciada destes profissionais. 
Para Fischer et al. (2017), proporcionar a reflexão bioética na sociedade, por meio do encontro entre profissionais de diferentes áreas, professores universitários, graduandos e estudantes do ensino básico, promove o crescimento moral de todos. A argumentação, a interação com a realidade, e o conhecer os valores dos outros possibilitam o alcance de soluções consensuais e justas, respondendo às necessidades do indivíduo, da humanidade, da sociedade, da natureza, desta e das próximas gerações (Fischer et al., 2017).

A bioética se relaciona à ideia de que é urgente, em todas as profissões, priorizar a formação integral da pessoa, preparando-a para se relacionar com os outros e com o mundo, convivendo e respeitando as diferenças, singularidades e opiniões (Wilges, 2007).

\section{Considerações Finais}

Cientistas da computação lidam direta ou indiretamente com atividades e desenvolvimento de tecnologias que afetam a vida e o modo de ser de pessoas, grupos culturais e da sociedade como um todo. Portanto, agem dotados de um componente moral, mesmo que inconsciente ou despercebido. A aproximação com a Bioética pode proporcionar aos futuros profissionais bases teóricas para uma reflexão qualificada e subsídios para a ação prudente, baseada no respeito e na compreensão das consequências atuais e futuras de suas decisões.

\section{Referências}

Adinolfi, V. T. S. (2014) "Educação em valores e bioética - formação de engenheiros". Tese (Doutorado) - Universidade de São Paulo, Faculdade de Educação, São Paulo.

ANSA, Brasil (2017) “Mega ataque cibernético atinge mais de 70 países”. Disponível em: http://ansabrasil.com.br. Acesso em 10 mai. 2018.

Beauchamp, T.; Childress, J. F. (2002) “Princípios de ética biomédica”. São Paulo: Loyola.

BSA (2018) "Business Software Alliance”. Disponível em: http://www.bsa.org. Acesso em: 10 mai. 2018.

Caramico, H. J; Zaher, V. L.; Rosito, M. M. B. (2007) "Ensino da bioética nas faculdades de medicina do Brasil". Biocthikos, v. 1, n. 1, p. 76-90.

Camargo, A.; Almeida, M. A. S.; Morita, I. (2014). "Ética e bioética: o que os alunos do sexto ano médico têm a dizer". Revista Brasileira de Educação Médica, v. 38, n. 2, p. 182-189.

Carneiro, Larissa A.; Porto, Celmo C.; Duarte, Soraya B. R.; Chaveiro, Neuma; Barbosa, Maria A. (2010). "O ensino da ética nos cursos de graduação da área de saúde”. Revista Brasileira de Educação Médica, v. 34, n. 3, p. 412-421.

Carvalho, Marcelo Sávio Revoredo Menezes de. (2006) “A trajetória da Internet no Brasil: do surgimento das redes de computadores à instituição dos mecanismo de governança. Dissertação (Mestrado) - Universidade Federal do Rio de Janeiro, COPPE, Engenharia de Sistemas e Computação, Rio de Janeiro. 
CFMV (2018) "IV Congresso Brasileiro de Bioética e Bem-Estar Animal”. Disponível em: http://bioeticaebea.cfmv.gov.br. Acesso em: 10 mai. 2018.

Cunha, T.; Lorenzo, C. (2014) "Bioética global na perspectiva da bioética crítica". Rev. bioét. (Impr.) v. 22, n. 1, p. 116-125.

Couto Filho, J. C. F; Souza, F. S.; Da Silva, S. S.; Yarid, S.; Sena, E. L. S. (2013) "Ensino da bioética nos cursos de Enfermagem das universidades federais brasileiras". Revista Bioética, v. 21, p. 179-185.

Dória, T. A. F.; Moreira, L. M. A. (2011) "A bioética na formação do biólogo: um desafio contemporâneo". Revista Entreideias: educação, cultura e sociedade, n. 20, p. 99-122.

Farinaccio, Rafael (2016) "Afinal, quanta energia elétrica a internet utiliza para funcionar?”. Disponível em: https://www.tecmundo.com.br. Acesso em: 10 mai. 2018.

Fischer, M. L.; Cunha, T. R.; Roth, M. E.; Martins, G. Z. (2017) "Caminho do Diálogo: uma experiência bioética no ensino fundamental”. Revista Bioética, v. 25, n. 1, p. 89100.

Loch, J. A. (2003) "Como analisar conflitos em bioética clínica”. In: Urban, C. A. Bioética clínica. Rio de Janeiro: Revinter, p. 48-54.

Mann, Ian (2011) "Engenharia Social - Série Prevenção de Fraudes". São Paulo: Blucher, $236 \mathrm{p}$.

Maluf, Fabiano; Azambuja, Letícia E. O. (2015) "Bioética e Odontologia: considerações sobre a relação profissional-paciente”. Revista Odontológica de Araçatuba, v. 36, n. 2, p. 61-65.

Mascarenhas, N. B.; Rosa, D. O. S. (2010) "Bioética e Formação do Enfermeiro: uma interface necessária". Texto \& Contexto - Enfermagem, v. 19, n. 2, p. 366-371.

MS (2018) "Ministério da Saúde - DATASUS - Meu digiSUS". Disponível em: https://play.google.com. Acesso em: 10 mai. 2018.

Neves Júnior, W. A.; Araújo, L. Z. S.; Rego, S. (2016) "Ensino de bioética nas faculdades de medicina no Brasil". Rev. Bioética v. 24, p. 98-107.

Otero, L. D. (2009) “Bioética: El concepto relegado”. Interciencia v. 34, n. 1, p. 7176.

Pereira, Bernardo Augusto da Costa (2015) "O Biodireito brasileiro, seus princípios e a Bioética". Revista Contribuciones a las Ciencias Sociales, n. 29.

Pessini, Leo. (2013) "As origens da bioética: do credo bioético de Potter ao imperativo bioético de Fritz Jahr”. Revista Bioética, v. 21, n. 1, p. 09-19.

Potter, V. R. (1970) "Bioethics: the science of survival". Persp Biol Med., v. 14, p. 127153.

Potter, V. R. (1971) "Bioethics: bridge to the future". Prentice Hall Inc. Englewood Cliffs New Jersey.

Potter, V. R. (1988) "Global bioethics: building on the Leopold legacy". East Lansing: Michigan State University Press. 
Potter, V. R. (1998) "Script do vídeo elaborado e apresentado para o IV Congresso Mundial de Bioética”. O Mundo da Saúde, v. 22, n. 6, p. 370-374.

Raymond, Gerson (2015) "Profissional de TI, Profissional Multitarefa ou Profissional Super-Homem?" Disponível em: https://www.tiespecialistas.com.br/2015/03/. Acesso em: 10 mai. 2018.

Roso, C. C.; Auler, D. (2016). “A participação na construção do currículo: práticas educativas vinculadas ao movimento CTS”. Ciência \& Educação (Bauru), v. 22, n. 2, p. 371-389.

Siebert, P. R. (2015) "Bioética para estudantes de Ciências Biológicas: investigação sobre os fundamentos que compõem ou deveriam compor a área”. Tese (Doutorado) - Universidade Estadual Paulista, Faculdade de Ciências, Bauru.

Silva, P. F.; Krasilchik, M. (2013) "Bioética e ensino de ciências: o tratamento de temas controversos - dificuldades apresentadas por futuros professores de ciências e de biologia”. Ciência \& Educação (Bauru), v. 19, n. 2, p. 379-392.

Siqueira-Batista, R; Gomes, A. P.; Maia, P. M.; Costa, I. T.; Paiva, A. O.; Cerqueira, F. R. (2014) "Modelos de tomada de decisão em bioética clínica: apontamentos para a abordagem computacional”. Revista Bioética, v. 22, n. 3, p. 456-461.

Siuly, S.; Huang, R.; Daneshmand, M. (2018) "Guest editorial: special issue on Artificial Intelligence in Health and Medicine". Health Information Science and Systems, v. 6.

Sitesa (2018) "Monitoramento de Empregados - Possibilidade". Disponível em: http://www.sitesa.com.br/contabil/conteudo_trabalhista/procedimentos/p_trabalhista/ m07.html. Acesso em: 10 mai. 2018.

Valladão, A. S. N.; Graciosa, L. K.; Silva, M. F.; Pecoraro, P. V. B. F. (2011) "A bioética odontológica contemporânea - ampliando concepções deontológicas”. Revista Interdisciplinar de Direito, Faculdade de Direito de Valença.

Villas-Bôas, M. E. (2012) "Bioética e direito: aspectos da interface". Desafios e perspectivas de um chamado biodireito. Revista Bioethikos, v. 6, n. 2, p. 89-100.

Zanetti, M. B. F. (2009) "O uso experimental de animais como instrumento didático nas práticas de ensino no curso de medicina veterinária". IX Congresso Nacional de Educação - EDUCERE. III Encontro Sul Brasileiro de Psicopedagogia, PUCPR. Curitiba.

Wilges, L. B. M. (2007) "A bioética num enfoque educacional: implicações na formação de professores de ciências e biologia”. Dissertação (Mestrado) - PUCRS, Pós-Graduação em Educação em Ciências e Matemática. 